\title{
NOTAS
}

\section{LA TRADICIÓN CORTESANA EN DOS COPLAS DE JUAN RUIZ}

Entre los muchos apóstrofes a la muerte que se encuentran en las literaturas europeas, merece especial atención el que compuso el Arcipreste de Hita con motivo de la muerte de Trotaconventos. Un planctus, por convencional que sea ${ }^{1}$, se convierte en algo distinto cuando lo elabora Juan Ruiz, sobre todo porque la víctima es una de esas viejas célebres por su conducta decididamente anticonvencional. De carácter insólito, en vista del modo como enfocan el concepto de la Muerte, son las coplas 1548-1549:

Tyras toda vergüença, desfeas fermosura, desadonas la graçia, denuestas la mesura, enflaquesçes la fuerça, enloquesçes cordura, lo dulce fazes fiel con tu much[a] amargura.

Despreçias loçanía, el oro escureçes, desfazes la fechura, alegría entristezes, manzyllas la lynpieza, cortesía envileçes, Muerte, matas la vida, al mundo aborresçes ${ }^{2}$.

Desde la primera lectura observamos elementos conocidamente cortesanos: alegría, cordura, cortesia, fermosura, vergüença (esto es 'modestia'), y asimismo los verbos desadonar, denostar, despreçiar. Habría que añadir expresiones como enflaquesçes la fuerça, el oro escureçes, desfazes la fechura, manzyllas la lynpieza, que a primera vista no parecen pertenecer a esta esfera. Un examen atento mostrará que las dos coplas constituyen un todo completo y que así pueden interpretarse, pues cada elemento se ajusta perfectamente en una de las divisiones del esquema cortesano. El dualismo implícito en las coplas por el hecho de tratarse de un postrer homenaje a la difunta Trotaconventos se explica como una de las muchas fantasías humorísticas del Arcipreste.

Muy escasa ayuda es la que nos ofrecen aquí los diccionarios corrientes. Sus definiciones son necesariamente incompletas, y no contienen los matices esenciales para la comprensión del concepto. El lector que encuentra, por ejemplo, la palabra cordura explicada como 'prudencia', 'juicio' se queda tan desorientado como antes, pues las palabras de la

${ }^{1}$ Cf. Libro de buen amor (Selección), ed., con estudio y notas, por María Rosa LidA, Buenos Aires, 1941, p. 159.

${ }^{2}$ Cito por la ed. de J. Ducamin, Toulouse-Paris, 1901, pero distingo la $z$ de la $s$ y corrijo acentuación y puntuación. 
definición necesitan ser interpretadas a su vez. Nuestro texto no es sencillo por sus ideas ni moderno en su expresión, de modo que hay que acoger con suma reserva el sentido que espontáneamente se le ocurre al lector del siglo xx. Así, pues, para estudiar las palabras hay que ver con qué términos se asocian de ordinario; hay que precisar la atmósfera en que florecen y la compañía en que suelen presentarse.

Son dos los aspectos que se ofrecen al investigador. Por una parte, hay que ver el sentido que la palabra tenía para el autor y sus contemporáneos, $\mathrm{y}$, por otra, observar su funcionamiento a la luz del estilo mismo del autor, particularmente dentro de los esquemas estructurales y de los métodos de yuxtaposición. Para examinar el primer aspecto procederemos por orden alfabético (es decir, arbitrario), y para estudiar el segundo haremos una recapitulación que muestre los matices y valores que adquieren las palabras en virtud de su concatenación en el texto ${ }^{3}$.

\section{1) Cordura}

La connotación más evidente del término es la de 'sentido común' o, como se diría hoy, 'equilibrio mental'. Así leemos en el Libro de buen amor: "el cuerdo con buen seso" (696a), "sey cuerdo e non sanudo, nin triste nin ayrado" $\left(5^{6}{ }_{3} c\right)$. Pero el último verso de la copla $5_{6}{ }_{3}$ muestra que esta idea ocupa un lugar especial dentro del esquema del poeta:

Sey como la paloma, linpio e mesurado,

sey commo el pavón, loçano, sosegado,

sey cuerdo e non sanudo, nin triste nin ayrado:

en [e]sto se esmera el que es enamorado.

Aparece en primer lugar la paloma, símbolo de la pureza, como expresamente lo dice el poeta. Lo que no dice, aunque sin duda lo sabía, es que la paloma es símbolo del Espíritu Santo, y que la mesura es uno de sus doce dones. La asociación linpio e mesurado no puede ser fortuita. Por lo demás, la paloma se propone como ejemplo a cualquier clase social, mientras que el pavón aparece en la procesión de infançones que forman el cortejo de don Carnal (1086ab):

Traýa buena mesnada rica de infançones:

muchos buenos faysanes, los loçanos pauones...

Penetramos así en la esfera cortesana, a la cual, como veremos, pertenece de manera eminente la palabra loçano. El hombre cuerdo, calificado por adjetivos adecuados (aunque no limitados a él), puede considerarse dentro de la escala así establecida, tanto más cuanto que ésta avanza de lo concreto - los dos primeros versos- a lo abstracto, en un movimiento que culmina en el último verso, según el cual la meta del enamorado es la distinción de un refinamiento especial ${ }^{4}$. Como ocurre a menudo en

s Aparte del interés puramente lexicográfico de este pasaje, conviene tener en cuenta que aqui se emplea la figura retórica de la expolitio, la cual es tan convencional como el contenido. La expolitio es un tipo de amplificatio por medio de repeticiones en forma de catálogo. Sobre estos procedimientos véase sobre todo E. Faral, Les arts poétiques $d u x^{i i^{\circ}}$ et $x i i i^{\circ}$ siècle, Paris, 1924 .

" No es difícil que el Arcipreste haya tenido conciencia de la etimología de esme- 
esta clase de términos, el lugar del concepto de cuerdo, dentro de la jerarquía del amor cortesano, roza las fronteras de la mística.

El antónimo de cuerdo es loco: "con los locos fácese loco, los cuerdos dél byen dixieron" $(728 c)$. En $1548 c$ hemos visto ya la combinación enloquesçes cordura. Lo importante es el antagonismo entre loco amor y buen amor, fundamental en toda la obra del Arcipreste. Existe, pues, una relación entre cordura y buen amor. El "amor guiado" de Avicena es, probablemente, el amor gobernado por el buen sentido ${ }^{5}$.

\section{Desadonas (la graçia)}

María Rosa Lida de Malkiel define 'descompones, quitas el donaire', y Cejador 'quitar el donaire y gracia'; existe, pues, una evidente relación entre desadonar y donaire. Covarrubias dice que tener gracia equivale a 'tener donaire y agrado', y a propósito de donaire afirma que "es lo mismo que don y gracia".

Spitzer ha hecho notar que la palabra donaire se relaciona con don 'regalo', y la interpreta 'don de plaire'6. Es importante este concepto, si recordamos la doctrina que ve en la gracia un don de Dios. Al igual que cordura, la palabra donaire es ambivalente, pues, como observa Américo Castro $^{7}$, se refiere tanto a lo mundano como a lo espiritual.

Para Spitzer, el sustantivo donaire (y el verbo correspondiente) es a todas luces un tópico cortesano, pues menciona un caso, rarísimo por cierto, en que donaire rima con el provenzal vejaire (Cancionero de Baena, núm. 240), asociación que supone una relación. Juan Ruiz, por supuesto, piensa sobre todo en el aspecto terrenal, esto es, en las gracias sociales; un hombre de tan evidente ortodoxia no podía decir que lo divino está sujeto, como lo humano, a los embates de la Muerte. Y sin embargo, no hay que tomar la palabra gracia (véase infra) en sentido demasiado material.

\section{3. (Desfazes la) fechura}

Fechura es más que el aspecto puramente exterior. En $1484 b c$ el Arcipreste emplea la palabra después de figura, término que debe tomarse en sentido más literal:

... que de ese arçipreste me digas su figura,

bien atal qual sea, dime toda su fechura,

rarse. La formación es bastante obvia, y mero como equivalente de 'puro' existía entonces como existe ahora. Por lo demás, si la forma reflexiva significa algo así como 'señalarse', 'distinguirse', la no reflexiva, según el Dicc. Aut., conserva su sentido etimológico.

${ }_{5}$ Cabría pensar que Avicena se hace eco, en su teoría, de ciertos conceptos platónicos. Véase A. J. Denomx, The heresy of courtly love, New York, 1947, p. 3o. Recuérdese el famoso Soneto $I_{3}$ de Du Bellay, con su "Au plus haut ciel guidée".

- "Notas etimológicas", RFE, 12 (1925), 235-237.

${ }^{7}$ España en su historia, p. 419. "Duplicidad de sentidos en el Libro de buen amor" es el título de uno de los capítulos. Juan Ruiz se complace maliciosamente en pasar de una esfera a la otra, sin situarse de plano él mismo en ninguna de ellas. "No trata de milagros sino de amor, ambiguo en sí mismo". No creo que haya necesidad de aducir aquí la doctrina de la "doble verdad". 
donde fechura se refiere a las cosas menos tangibles, las que están más allá del aspecto personal. En este sentido, la palabra se asemeja al ant. prov. faisson, que Crescini ha definido impropiamente 'fazzone, forma, figura, manera'. Juan Ruiz no habría confundido estas palabras, puesto que separa claramente figura de fechura, que es la que equivale a 'manera' o 'fazzone'. En ant. fr. faiture es término elogioso, empleado, por ejemplo, en la evocación de la belleza de Alcibíades (Roman de la Rose, ed. Langlois, vs. 8943 ss.):

car le cors Alcibiadès

que de beautez avoit adès, et de couleur e de faiture...

La definición que de esta palabra da Grandsaignes d'Hauterive - 'allure, prestance'- le añade la idea de cierta nobleza de porte, de modo que no resulta extraño encontrar en Jean Lemaire (HUGuET, $s$. v.) el grupo noble faiture.

En Juan Ruiz, como tantas veces ocurre, el aspecto físico es paralelo al valor espiritual. Al destruir el primero, la Muerte aniquila también el segundo.

El verbo no ofrece aquí especial interés, excepto por el hecho de tener la misma raíz que el sustantivo, según el conocido procedimiento medieval de la deriuatio.

\section{4. (Desfeas) fermosura}

En la poesía provenzal, la idea de belleza va mucho más allá de lo material; cf. Guilhelm IX (ed. Jeanroy), "Mout jauzens...", estr. 5:

Per son joy pot malautz sanar,

$e$ per sa ira sas morir

$e$ savis hom enfolezir

$e$ belhs hom sa beutat mudar

$e \cdot l$ plus cortes vilanejar

$e$ totz vilas encortezir.

Beutat aparece entre savis y cortes; la pérdida de la beutat es análoga a la antítesis savis / enfolezir o vilas / encortezir. Juan Ruiz debe de haber pensado lo mismo al escribir la copla 1613:

Como robý pequeño tyene mucha bondat, color, virtud e preçio e noble claridad, ansý dueña pequeña tiene mucha beldat, fermosura, donayre, amor e lealtad.

Amor y lealtad no requieren comentario. El donayre, como hemos visto, es algo más que porte cortesano. La fermosura, asociada con esos tres conceptos en el último verso, se relaciona, algo remotamente, con bondat, cosa nada rara si se tiene en cuenta el fermosura e bomdade portugués (Nunes, Crestomatia arcaica, p. 21) y la identificación de bellezza con vertute en Petrarca (Canzoniere, 337, v. 6, ed. Scherillo, Milano, 1925). Por supuesto, el poeta del Libro de buen amor no penetra en las honduras del misticismo, pero no podía escapársele la especial aplicación del 
concepto de belleza en la esfera espiritual. Recuérdese el Merito praedicatur pulchra... (PL, t. 196, col. 482) de Ricardo de San Víctor, y las palabras de Santo Tomás (Summa theologica, Secunda secundae, q. 116, art. 2): "La belleza del cuerpo consiste en la buena proporción de los miembros corporales del hombre, junto con cierta claridad del color. Del mismo modo, la belleza espiritual consiste en una buena proporción de la conducta o las acciones del hombre con respecto a la claridad espiritual de la razón". Como se ve, ni siquiera los teólogos separan un nivel del otro. Arnaldo de Villanova califica la bellezza de "virtù corporale" y la coloca al lado de la ioventudine, la forteza, la gientilezza y la signioria, opuestas a la "gratia spirituale" 8 . Para el hombre medieval no hay nada raro en tales ambivalencias.

\section{5. (Enflaquesçes) la fuerça}

La idea misma de fuerza no supone connotaciones extrañas. Pero, como acabamos de ver, Arnaldo de Villanova incluye la forteza entre las "virtudes" propias del cortesano. Ésa es también la asociación que establece Juan Ruiz (245cd) :

que fuerça e hedat e onrra, salud e valentía non pueden durar syenpre: vanse con mançebía.

El primer término queda relacionado con onrra en un nivel superior, pero por otra parte toda la serie está bajo el signo de lo transitorio, esto es, de lo que los trovadores llamaban joven. Hay casos en que la fuerza se asocia con una abstracción de tipo más elevado, sin que el poeta tenga conciencia de estar reuniendo elementos heterogéneos, por ejemplo Robert de Blois, Enseignement des princes, vs. 1101-1102: “...doners est grace / sor savoir, sor force et bonté". Sería extraño encontrar aquí force entre savoir y bonté si no fuera porque la palabra aparece, insólitamente, como atributo personal, con connotaciones más que físicas.

Es decir, la Muerte no sólo aniquila las facultades corporales - cosa sabida-, sino con ellas un rasgo del carácter.

\section{6. (Desadonas) la graşia}

Ya hemos hablado de esta expresión a propósito del verbo. Las asociaciones del sustantivo merecen atención especial. En la medida en que se trata de "gracias sociales", no hay nada inesperado; pero se puede llegar con facilidad a un nivel superior, como en estos versos de García de Borja (Cancionero de Palacio, núm. 256, vs. 1-2):

Ioventut, gracia et calor, d'amor mis mantenedores,

donde calor aleja a gracia del ámbito puramente físico y, además, los tres atributos vienen a ser "mantenedores" del amor. Es algo más sorprendente encontrar una pareja como gracia y saber (ibid., núm. 340, v. 11), o ver que es posible ser al mismo tiempo óme gracioso e leal (Kalila

${ }^{8}$ Cf. R. Manselli, La religiosità d'Arnaldo de Villanova, Roma, 1951, p. 64. 
et Digna) ${ }^{9}$. Muy notable es el hecho de que Pedro de Santa Fe, en el mismo Cancionero de Palacio (núm. 312, vs. 10-14), asocie tres términos agrupados igualmente en las coplas de Juan Ruiz:
si vos lohan de cordura
no mienten, pues en vos cabe
aún gracia e fermosura;
pues menos dirá locura
quien vos lohe de franqueza.

No hay para qué explicar la asociación con cordura. También Chaucer tiene un concepto elevado de la gracia cuando habla de "largesse and all grace" (Complainte of fair Anelida, 42), pero no concuerda exactamente con la categoría de la palabra en español.

\section{7. (Manzyllas) la lynpieza}

Después de las dos coplas que venimos estudiando, Juan Ruiz no vuelve a emplear el esquema verbo-sustantivo, típico de la anterior serie de expresiones, excepto en la copla ${ }_{1555}$ :

Tú despoblaste, Muerte, al çielo e sus syllas,

los que eran lynpieça fezístelos manzillas.

Por supuesto, el poeta dice esto en sentido figurado. Chaucer emplea del mismo modo la palabra clennesse 'limpieza' en el v. 465 de The Parson's tale: "Usynge vertu, curtesye and clennesse", combinación extraña, pues curtesye aparece entre dos términos que están más de acuerdo con el lenguaje de un clérigo.

La palabra limpieza tiene gran número de connotaciones. El Dicc. Aut. trae 'aseo, curiosidad, castidad, pureza, integridad, desinterés'. Tanto los sentidos literales como los figurados pertenecen al mundo cortesano, en el cual siempre tuvo un lugar la limpieza física al lado de la pureza moral. El equivalente francés netée tiene un sentido especial: "Tuilles dit, ta netées doit estre tele k'ele ne soit haïe par trop aornement, mais tant que tu ostes la sauvage negligence et sa campestre laidece" (BRUnetto Latini, Tresor, ed. Carmody, p. 250), donde netée equivale a un despojarse de los rasgos indeseables del carácter o de la conducta. Es significativo que este pasaje del Tresor se encuentre en un capítulo consagrado a la Mesura. No es raro, pues, que la limpieza se asocie con la franqueza:

çimiento de la franqueza, metal de toda linpieza,

dice una Copla del Condestable que fizo al Rey de Castilla (Cancionero de Palacio, núm. $4^{8}$, vs. $5^{-6}$ ). De este aspecto se ocupan aun algunos escritores místicos, como Ricardo de San Víctor (Exegetica explicatio in Cantica canticorum, en $P L$, t. 196, col. 463): "Et quot superfluis verbis maculas contraxerit, tot de gratiae dulcedine exsiccabit. Per oris ergo custodiam servat cordis munditiam, et ex cordis munditia foris format verba".

- R. M. PÉrez, Vocabulario clasificado de "Kalila et Digna" (tesis), Chicago, 1943, p. 95 . 
Así, pues, para volver a Juan Ruiz, la Muerte no sólo mancha y desprecia aquello que es limpio de cuerpo, sino hasta lo que es puro de corazón.

\section{8. (Despreçias) loçania}

Es evidente la relación con la esfera cortesana: "muy loçano e cortés, sobre todos esmerado" $(1327 d)$ y, en seguida, con una cualidad cortesana como la belleza, según se observa en una antigua paráfrasis portuguesa del Quam pulchra es, donde se emplea varias veces esta pareja de sinónimos: "cam fremosa es e cam louçãa" (NunEs, Crestomatia arcaica, p. 63).

Es importante el matiz de sosiego: "sey commo el pavón, loçano, sosegado" $\left(5^{6} 3^{b}\right)$. La diferencia entre loçano y una palabra como donaire, referida al aspecto exterior, queda patente en el contraste: "oras triste, sanudo, oras seýe loçano" (1279b); de la monja en oración dice igualmente el Arcipreste que es loçana $\left(1499^{b}\right)$.

No falta un matiz de orgullo, pues Aguado traduce enloçanecer por 'ufanarse'. Pero esta ufanía, que a los ojos de todos se presenta como un valor auténtico, no hace mella en la Muerte, la cual priva inexorablemente al individuo de aquello que los trovadores provenzales llamaban pretz ('valor, estimación'). Este pretz está justamente en la base de despreciar. A la Muerte no le importa el valor convencional que la sociedad cortesana atribuye a la presencia y a las cualidades externas, ni siquiera cuando se trata de los servidores del buen amor. La Muerte menosprecia tales cosas, así como menosprecia el oro, que a sus ojos pierde brillo y se opaca. (cf. el oro escureces).

\section{Vergüença}

Aguado extiende el sentido de la palabra al 'color sonrosado, signo de ese poder', pero añade en seguida la expresión más abstracta omne de vergüença 'esto es: de pundonor'. La palabra pundonor aproxima la idea al ámbito del buen amor, pues está en relación con el Dangier del Roman de la Rose, donde la modestia desempeña un papel tan importante. Esto determina el sentido de tirar, que sugiere un descorrer el velo de la reserva. Así como despreçias loçanía tiene su complemento en el hemistiquio siguiente, el oro escureçes, así también tyras toda vergüença se completa con desfeas fermosura. Al descorrerse el velo, no se muestra la belleza, sino que se desvalora algo que sólo tiene valor cuando lo rodea la reserva. En el mundo árabe sobre todo - aun dentro de la relativa libertad del régimen social andaluz-, la belleza no estaba hecha primordialmente para exhibirse. Pero, como observa el Arcipreste, la Muerte no respeta tales consideraciones ${ }^{10}$.

Hasta aquí hemos examinado las diversas expresiones desde el punto de vista lexicográfico, sin darles más contexto que un solo verso. Pero

10 Sobre el contenido semántico de vergüenza ha influído en no poca medida el pensamiento árabe, a juzgar por lo que dice CASTro, op. cit., p. 69. 
no debemos limitarnos a esa fragmentación, pues estamos en presencia de un artista, esto es, de un hombre que no puede menos que crear una estructura literaria. Tenemos que considerar las dos coplas como un todo.

Este procedimiento resulta tanto más necesario, cuanto que la yuxtaposición de los tópicos incluídos en las coplas no es mera coincidencia, como lo muestra el siguiente pasaje ("Macías", en el Cancionero de Palacio, núm. 291, estr. 2):

Con él venía mesura,
la muy gentil cortesia,
poderosa cordura,
briosa locania;
regláualos fermosura,
que traýa gran valor
con que amor
vencía la mi cordura.

Cinco de los términos empleados por el Arcipreste aparecen aquí en otros tantos versos.

En el Libro de buen amor hay un escalonamiento de ideas que podría parafrasearse del modo siguiente: Lo primero que hace la muerte es descorrer el velo de la reserva, pues no la respeta. Sin ese respeto de sí misma, representado por la vergüença, carece de valor la belleza, y de interés la búsqueda de la belleza. La gracia física, corolario de la fermosura, pierde su calidad de don en cuanto ésta desaparece. Toda la estructura moral del individuo se desequilibra. Sabemos la importancia que tiene la mesura en la concepción del héroe épico, y sabemos también que la falta de equilibrio puede ser trágica, pues conduce a la deshonra o a una catástrofe física total. Una vez producido el desequilibrio, el individuo no tiene ya verdadera fuerza, ni física ni moral (ambas son inseparables) ; el cobarde del Couronnement de Louis carece de vigor corporal y de fortaleza de espíritu. Esta interpretación explica la intercalación, extraña a primera vista, de enflaquesçes la fuerça entre las palabras mesura y cordura. Ya hemos hablado sobre el grado de abstracción de cordura, equivalente al buen de buen amor. Podemos decir, pues, que, pasando de un aspecto al otro, destruyendo la reserva, quitando toda la gracia y debilitando la fuerza que trae consigo la hermosura, la Muerte logra desintegrar la personalidad y hace desaparecer el equilibrio y la mesura, hasta el punto de que la cordura cede a la locura. En la culminación de esta primera copla, el poeta no puede menos que exclamar: Ahora todo es amargo.

En la segunda copla es más difícil, aunque no imposible, seguir la concatenación de ideas. De nuevo se nos aparece la Muerte en su tarea destructora de la dignidad humana. Todo pierde su valor: el oro se opaca; la presencia exterior del hombre no puede mantenerse. Por otra parte, se convierte en tristeza la alegria, palabra que no sólo significa el gozo ordinario, sino algo así como la exultación del trovador, que permite al hombre llegar hasta la pureza del corazón, la lynpieza. Se ha sugerido - y Castro lo ha probado- que esta palabra llega a tener posibilidades teológicas. Pero el caprichoso Arcipreste, que teje una compleja y 
delicada transición entre lo profano y lo espiritual, coloca la lynpieza inmediatamente después de la cortesía, la cual - cosa significativa- constituye la culminación de las dos coplas. Extinguida la cortesía, el poeta se ve forzado a reconocer: "Muerte, matas la vida, al mundo aborresçes". Las cualidades que integran el "buen amor" han quedado aniquiladas una tras otra en la muerte de la vieja. El poeta, con ademán de grandilocuente desesperación, renuncia a seguir por este camino, y desde este momento tomará rumbos diversos.

The Ohio State University.

A. H. Schutz

\section{GLORIA}

Aunque las abundantísimas expresiones españolas de origen religioso proceden de diversas fuentes -el santoral, las actividades de la Iglesia, los escritos de los Padres, etc.-, las más tienen su fuente en la liturgia. La misa, las letanías y los sacramentos, que todos los católicos conocen, les ofrecen la tentación continua de servirse de su lenguaje, de modo que existe en español una larga lista de palabras litúrgicas cuya historia valdría la pena investigar.

Uno de los términos más interesantes de este grupo es gloria. En la misa aparece primero en el Gloria Patri, cantado después del salmo Iudica me:

Gloria Patri, et Filio, et Spiritui Sancto.

Sicut erat in principio, et nunc, et semper, et in saecula saeculorum.

La explicación de esta palabra trae consigo gran número de problemas. Gloria, además de ser término cristiano, se encuentra en la literatura profana, con varios significados. El Harper's Latin Dictionary nos informa que, en latín clásico, gloria significaba 'gloria', 'fama', 'renombre', 'elogio', 'honor', 'ornamento', 'orgullo', 'afán de gloria', etc. Los mismos sentidos se encuentran en castellano, además de otros que provienen del uso eclesiástico. Los significados actuales de gloria en la Iglesia católica ('esplendor', 'ostentación', etc.) se deben al uso de gloria en la Biblia latina y en los Padres de la Iglesia. Éstos traducían con gloria la palabra $\delta o ́ \xi a$, que a su vez había recibido su connotación del hebreo. La "gloria" de Salomón -es decir, el esplendor de su corte- y la "gloria" de Dios - la majestad divina - son conceptos bíblicos, no clásicos. Bíblica es también la significación implícita en "Gloria a Dios", donde gloria equivale a 'homenaje'1. El estado de los bienaventurados era llamado gloria por los escritores eclesiásticos de la Edad Media, sin duda a causa de su esplendor sobrehumano. En el Catecismo del P. Ripalda hallamos: "Los novísimos o postrimerías del hombre son cuatro: muerte, juicio, infierno y gloria". Podemos definir gloria como 'brillo, esplendor, majestad', 'la presencia de Dios'.

1 Cf. Hans Rheinfelder, Kultsprache und Profansprache in den romanischen Ländern, Genève-Firenze, 1933, pp. 282 ss. 